11 *Corresponding author footnote

12 4-10 Ag/For Centre

13 Edmonton, AB T6E2P5, Canada

14 Phone:+1 7804923634

$15 \quad$ Fax: +7804924265

16 Email: mgaenzle@ualberta.ca

\section{P.R. China}

17 
20 Table S1. Cell counts and $\mathrm{pH}$ sourdoughs fermented by different strains for $96 \mathrm{~h}$.

21 Table S2. Concentration of $\gamma$-glutamyl dipeptides ( $\mu \mathrm{mol} / \mathrm{kg})$ in rye-malt sourdoughs fermented 22 with L. reuteri $100-23$ or $100-23 \Delta \operatorname{gadB}$ and addition or protease, or not.

Table S3. Concentration of $\gamma$-glutamyl dipeptides $(\mu \mathrm{mol} / \mathrm{kg})$ in rye or rye-malt sourdoughs fermented with $L$. reuteri $100-23$ or $100-23 \Delta \operatorname{gadB}$ and different ingredients

25

Figure S1. Free amino nitrogen of sourdoughs fermented by L. reuteri 100-23 (black bars) and L. reuteri 100-23 $\Delta$ gadB (grey bar) for 48h (Panel A) and 96h (Pane B). Rye malt or whole rye flour was used as fermentation substrate as indicated; rye malt sourdoughs additionally contained protease and / or transglutaminase as indicated. Data represents means \pm standard deviations of duplicate independent experiments that were analysed with technical repeats. Values in the same panel that do not share a common superscript differ significantly $(\mathrm{p}<0.05)$. 
Table S1. Cell counts and pH sourdoughs fermented by different strains for $96 \mathrm{~h}$.

\begin{tabular}{|c|c|c|c|c|c|}
\hline & TMW 1.106 & LTH5448 & $100-23$ & $100-23 \Delta \operatorname{gadB}$ & $\begin{array}{c}\text { Chemically } \\
\text { acidified }\end{array}$ \\
\hline \multicolumn{6}{|c|}{ pH } \\
\hline $\mathbf{O} \mathbf{h}$ & $6.05 \pm 0.02$ & $6.095 \pm 0.02$ & $6.095 \pm 0.06$ & $6.10 \pm 0.02$ & $3.38 \pm 0.03$ \\
\hline $24 \mathrm{~h}$ & $3.57 \pm 0.02$ & $3.55 \pm 0.01$ & $3.6 \pm 0.00$ & $360 \pm 0.01$ & $3.65 \pm 0.02$ \\
\hline $48 \mathrm{~h}$ & $3.46 \pm 0.12$ & $3.61 \pm 0.02$ & $3.65 \pm 0.01$ & $3.66 \pm 0.01$ & $3.99 \pm 0.02$ \\
\hline $72 \mathrm{~h}$ & $3.71 \pm 0.02$ & $3.72 \pm 0.00$ & $3.735 \pm 0.01$ & $3.71 \pm 0.00$ & $3.77 \pm 0.01$ \\
\hline $96 \mathrm{~h}$ & $3.81 \pm 0.03$ & $3.83 \pm 0.03$ & $3.79 \pm 0.01$ & $3.80 \pm 0.02$ & $3.97 \pm 0$ \\
\hline \multicolumn{6}{|c|}{ cell count $(\mathrm{cfu} / \mathrm{g})$} \\
\hline $\mathbf{O} \mathbf{h}$ & $1.00 \times 10^{7}$ & $1.00 \times 10^{7}$ & $1.00 \times 10^{7}$ & $1.00 \times 10^{7}$ & $1.00 \times 10^{3}$ \\
\hline $24 \mathrm{~h}$ & $1.80 \times 10^{9}$ & $1.90 \times 10^{9}$ & $1.75 \times 10^{9}$ & $8.90 \times 10^{8}$ & $1.00 \times 10^{3}$ \\
\hline $48 \mathrm{~h}$ & $5.95 \times 10^{8}$ & $5.20 \times 10^{8}$ & $2.05 \times 10^{8}$ & $3.95 \times 10^{8}$ & $7.50 \times 10^{4}$ \\
\hline $72 \mathrm{~h}$ & $2.20 \times 10^{9}$ & $1.45 \times 10^{9}$ & $1.50 \times 10^{8}$ & $6.40 \times 10^{8}$ & $7.50 \times 10^{4}$ \\
\hline $96 \mathrm{~h}$ & $6.40 \times 10^{8}$ & $7.33 \times 10^{8}$ & $6.10 \times 10^{8}$ & $4.45 \times 10^{7}$ & $1.00 \times 10^{4}$ \\
\hline
\end{tabular}


Table S2. Concentration of $\gamma$-glutamyl dipeptides $(\mu \mathrm{mol} / \mathrm{kg}$ ) in rye malt sourdough fermented

37 with L. reuteri $100-23$ or L. reuteri $100-23 \Delta \operatorname{gadB}$ and protease addition, or not.

\begin{tabular}{|c|c|c|c|c|c|c|}
\hline & $\gamma$-Glu-Glu & $\gamma$-Glu-Ile & $\gamma$-Glu-Leu & $\gamma$-Glu-Met & $\gamma$-Glu-Phe & $\gamma$-Glu-Val \\
\hline \multicolumn{7}{|c|}{ L. reuteri $100-230 \mathrm{~h}$} \\
\hline $\begin{array}{c}\text { Rye malt+ } \\
\text { Fungal protease }\end{array}$ & $7.4 \pm 6^{c}$ & $1.6 \pm 1.6^{\mathrm{bc}}$ & $3.0 \pm 3.0^{\mathrm{a}}$ & $1.7 \pm 0.0^{\mathrm{f}}$ & nd & nd \\
\hline Rye malt & $9.6 \pm 0.4^{\mathrm{b}}$ & $2.1 \pm 0.2^{\mathrm{c}}$ & $3.1 \pm 3.1^{\mathrm{a}}$ & $1.7 \pm 0.0^{\mathrm{f}}$ & nd & $0.4 \pm 0.5^{\mathrm{c}}$ \\
\hline \multicolumn{7}{|c|}{ L. reuteri $100-2348 \mathrm{~h}$} \\
\hline $\begin{array}{c}\text { Rye malt+ } \\
\text { Fungal protease }\end{array}$ & $14.5 \pm 1.1^{\mathrm{a}}$ & $7.2 \pm 0.9^{\mathrm{a}}$ & $3.7 \pm 1.2^{\mathrm{a}}$ & $5.1 \pm 0.5^{\mathrm{a}}$ & nd & $1.7 \pm 1.2^{\mathrm{bc}}$ \\
\hline Rye malt & $9.7 \pm 0.5^{\mathrm{b}}$ & $1.8 \pm 0.7^{\mathrm{bc}}$ & $4.5 \pm 0.2^{\mathrm{a}}$ & $4.2 \pm 0.3^{\mathrm{b}}$ & nd & $1.3 \pm 0.1^{\mathrm{b}}$ \\
\hline \multicolumn{7}{|c|}{ L. reuteri $100-2396 \mathrm{~h}$} \\
\hline $\begin{array}{c}\text { Rye malt+ } \\
\text { Fungal protease }\end{array}$ & $10.2 \pm 1.2^{\mathrm{b}}$ & $2.46 \pm 0.7^{c}$ & $4.3 \pm 0.0^{\mathrm{a}}$ & $4.5 \pm 0.2^{\mathrm{b}}$ & $0.01 \pm 0.00$ & $4.6 \pm 0.6^{\mathrm{a}}$ \\
\hline Rye malt & $9.8 \pm 1.9^{b}$ & $\mathrm{Nd}$ & $2.3 \pm 1.0^{\mathrm{a}}$ & $3.2 \pm 0.3^{\mathrm{c}}$ & nd & $1.6 \pm 2.5^{\mathrm{bc}}$ \\
\hline \multicolumn{7}{|c|}{ L. reuteri $100-23 \Delta \operatorname{gadB} 0 \mathrm{~h}$} \\
\hline $\begin{array}{c}\text { Rye malt+ } \\
\text { Fungal protease }\end{array}$ & $8.7 \pm 0.7^{b}$ & $1.6 \pm 0.4^{\mathrm{bc}}$ & $3.4 \pm 3.4^{\mathrm{a}}$ & $1.7 \pm 0.0^{\mathrm{e}}$ & nd & $1.2 \pm 1.7^{\mathrm{bc}}$ \\
\hline Rye malt & $9.1 \pm 0.5^{\mathrm{b}}$ & $5.2 \pm 0.3^{\mathrm{a}}$ & $2.6 \pm 2.6^{\mathrm{a}}$ & $1.7 \pm 0.0^{\mathrm{e}}$ & nd & $0.3 \pm 0.4^{\mathrm{c}}$ \\
\hline \multicolumn{7}{|c|}{ L. reuteri $100-23 \Delta \operatorname{gadB} 48 \mathrm{~h}$} \\
\hline $\begin{array}{c}\text { Rye malt+ } \\
\text { Fungal protease }\end{array}$ & $10.3 \pm 3.3^{b}$ & $5.7 \pm 3.1^{\mathrm{a}}$ & $6.5 \pm 0.3^{\mathrm{a}}$ & $3.4 \pm 1.0^{\mathrm{c}}$ & nd & $0.3 \pm 0.6^{c}$ \\
\hline Rye malt & $10.5 \pm 0.9^{\mathrm{b}}$ & $0.2 \pm 0.2^{\mathrm{c}}$ & $6.6 \pm 0.8^{\mathrm{a}}$ & $1.6 \pm 0.0^{\mathrm{e}}$ & nd & $1.6 \pm 0.1^{\mathrm{b}}$ \\
\hline \multicolumn{7}{|c|}{ L. reuteri $100-23 \Delta \operatorname{gadB} 96 \mathrm{~h}$} \\
\hline $\begin{array}{c}\text { Rye malt+ } \\
\text { Fungal protease }\end{array}$ & $14.6 \pm 1.2^{\mathrm{a}}$ & $2.7 \pm 1.1^{\mathrm{b}}$ & $5.2 \pm 1.6^{\mathrm{a}}$ & $3.2 \pm 0.9^{c}$ & $0.01 \pm 0.00$ & $1.0 \pm 0.8^{\mathrm{bc}}$ \\
\hline Rye malt & $12.6 \pm 3.1^{\mathrm{a}}$ & nd & $4.3 \pm 0.0^{\mathrm{a}}$ & $2.2 \pm 0.7^{\mathrm{d}}$ & nd & nd \\
\hline
\end{tabular}

38 Data represent means \pm standard deviations of duplicate independent experiments analysed in

39 duplicate. Data in the same column that do not share a common superscript differ significantly.

40 nd, not detected. 
42 Table S3. Concentration of $\gamma$-glutamyl dipeptides $(\mu \mathrm{mol} / \mathrm{kg})$ in sourdough fermented with 43 different ingredients.

\begin{tabular}{|c|c|c|c|c|c|c|}
\hline & $\gamma$-Glu-Glu & $\gamma$-Glu-Ile & $\gamma$-Glu-Leu & $\gamma$-Glu-Met & $\gamma$-Glu-Phe & $\gamma$-Glu-Val \\
\hline \multicolumn{7}{|c|}{ L.reuteri $100-230 \mathrm{~h}$} \\
\hline $\begin{array}{c}\text { Rye malt+ Fungal } \\
\text { protease+MTG }\end{array}$ & $6.72 \pm 2.08^{\mathrm{d}}$ & $0.91 \pm 0.91^{\mathrm{c}}$ & $3.78 \pm 3.78^{\mathrm{a}}$ & $1.78 \pm 0.11^{\mathrm{cd}}$ & nd & nd \\
\hline $\begin{array}{c}\text { Rye }+ \text { Fungal } \\
\text { protease+MTG }\end{array}$ & $9.33 \pm 0.10^{c}$ & nd & $1.61 \pm 1.61^{\mathrm{a}}$ & $1.68 \pm 0.01^{\mathrm{d}}$ & nd & $0.72 \pm 0.10$ \\
\hline \multicolumn{7}{|c|}{ L.reuteri 100-23 $48 \mathrm{~h}$} \\
\hline $\begin{array}{c}\text { Rye malt+ Fungal } \\
\text { protease+MTG }\end{array}$ & $8.04 \pm 0.13^{\mathrm{d}}$ & $3.08 \pm 1.99^{b}$ & $4.17 \pm 0.61^{\mathrm{a}}$ & $3.15 \pm 0.58^{\mathrm{a}}$ & $0.07 \pm 0.01$ & $0.98 \pm 0.50$ \\
\hline $\begin{array}{c}\text { Rye }+ \text { Fungal } \\
\text { protease+MTG }\end{array}$ & $9.68 \pm 0.31^{c}$ & $6.78 \pm 0.63^{a}$ & $3.04 \pm 0.65^{\mathrm{a}}$ & $2.51 \pm 0.14^{\mathrm{bc}}$ & nd & $4.87 \pm 3.89$ \\
\hline \multicolumn{7}{|c|}{ L.reuteri 100-2396 h } \\
\hline $\begin{array}{l}\text { Rye malt+ Fungal } \\
\text { protease+MTG }\end{array}$ & $8.02 \pm 0.99^{d}$ & $0.64 \pm 0.64^{\mathrm{c}}$ & $4.52 \pm 0.15^{\mathrm{a}}$ & $2.77 \pm 0.24^{\mathrm{b}}$ & $0.07 \pm 0.01$ & $1.78 \pm 0.22$ \\
\hline $\begin{array}{c}\text { Rye }+ \text { Fungal } \\
\text { protease+MTG }\end{array}$ & $9.68 \pm 0.88^{c}$ & $1.93 \pm 0.44^{b c}$ & $3.60 \pm 0.51^{\mathrm{a}}$ & $2.37 \pm 0.04^{\mathrm{bc}}$ & nd & $3.95 \pm 4.30$ \\
\hline \multicolumn{7}{|c|}{ L.reuteri $100-23 \Delta$ gadB $0 \mathrm{~h}$} \\
\hline $\begin{array}{c}\text { Rye malt+ Fungal } \\
\text { protease+MTG }\end{array}$ & $5.99 \pm 2.14^{\mathrm{d}}$ & $0.97 \pm 0.97^{\mathrm{c}}$ & $3.90 \pm 3.90^{\mathrm{a}}$ & $1.69 \pm 0.01^{\mathrm{d}}$ & nd & $0.76 \pm 1.07$ \\
\hline $\begin{array}{c}\text { Rye }+ \text { Fungal } \\
\text { protease+MTG }\end{array}$ & $7.65 \pm 0.27^{\mathrm{d}}$ & nd & $1.58 \pm 1.58^{\mathrm{a}}$ & $1.68 \pm 0.01^{\mathrm{d}}$ & nd & $0.42 \pm 0.60$ \\
\hline \multicolumn{7}{|c|}{ L.reuteri $100-23 \Delta \operatorname{gadB} 48 \mathrm{~h}$} \\
\hline $\begin{array}{l}\text { Rye malt+ Fungal } \\
\text { protease+MTG }\end{array}$ & $8.95 \pm 0.11^{\mathrm{c}}$ & $4.97 \pm 1.64^{\mathrm{a}}$ & $5.89 \pm 1.56^{\mathrm{a}}$ & $3.20 \pm 0.03^{\mathrm{a}}$ & $0.08 \pm 0.01$ & $0.51 \pm 0.07$ \\
\hline $\begin{array}{c}\text { Rye }+ \text { Fungal } \\
\text { protease+MTG }\end{array}$ & $8.55 \pm 0.15^{\mathrm{d}}$ & $5.97 \pm 0.17^{\mathrm{a}}$ & $3.50 \pm 0.28^{\mathrm{a}}$ & $2.42 \pm 0.22^{\mathrm{bc}}$ & nd & $1.45 \pm 0.28$ \\
\hline \multicolumn{7}{|c|}{ L.reuteri $100-23 \Delta \operatorname{gadB} 96 \mathrm{~h}$} \\
\hline $\begin{array}{l}\text { Rye malt+ Fungal } \\
\text { protease+MTG }\end{array}$ & $13.17 \pm 4.95^{b}$ & $0.01 \pm 0.01^{\mathrm{c}}$ & $3.33 \pm 1.58^{\mathrm{a}}$ & $2.56 \pm 0.32^{\mathrm{b}}$ & nd & nd \\
\hline $\begin{array}{c}\text { Rye }+ \text { Fungal } \\
\text { protease+MTG }\end{array}$ & $13.17 \pm 4.95^{\mathrm{a}}$ & $1.00 \pm 0.45^{\mathrm{c}}$ & $1.29 \pm 0.40^{\mathrm{a}}$ & $2.45 \pm 0.12^{\mathrm{bc}}$ & nd & $0.89 \pm 1.26$ \\
\hline
\end{tabular}

44 Data represents means \pm standard deviations of duplicate independent experiments that were 45 analysed with technical repeats. Values in the same column that do not share a common

46 superscript differ significantly $(p<0.05)$

47 MTG, microbial transglutaminase; nd, not detected 


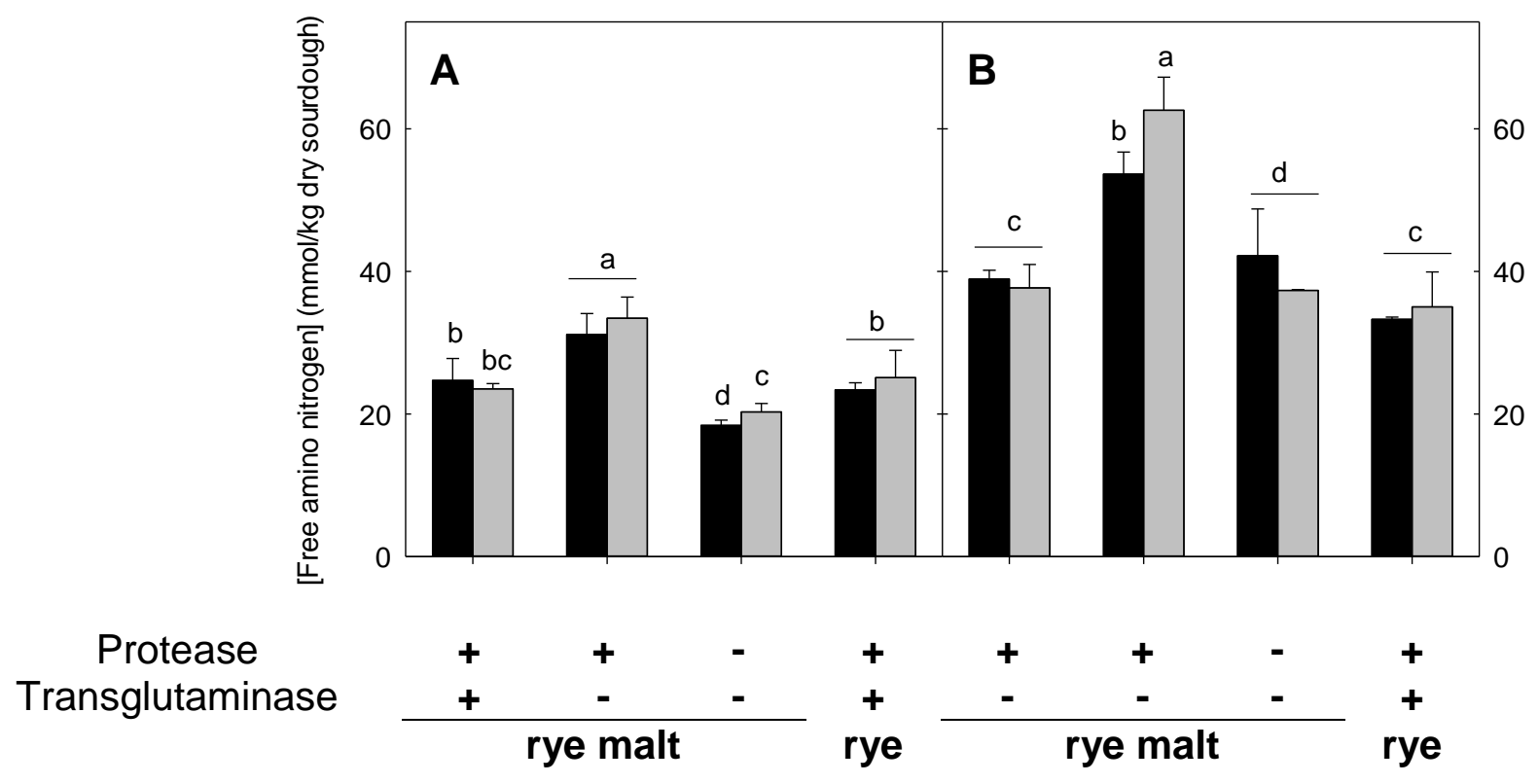

50

51 Figure S1. Free amino nitrogen of sourdoughs fermented by L. reuteri 100-23 (black bars) and

52 L. reuteri 100-23 $\triangle$ gadB (grey bar) for $48 \mathrm{~h}$ (Panel A) and 96h (Pane B). Rye malt or whole rye

53 flour was used as fermentation substrate as indicated; rye malt sourdoughs additionally contained

54 protease and / or transglutaminase as indicated. Data represents means \pm standard deviations of 55 duplicate independent experiments that were analysed with technical repeats. Values in the same 56 panel that do not share a common superscript differ significantly $(\mathrm{p}<0.05)$. 